\title{
Concentration of mass on convex bodies
}

\author{
G. Paouris*
}

\begin{abstract}
We establish a sharp concentration of mass inequality for isotropic convex bodies: there exists an absolute constant $c>0$ such that if $K$ is an isotropic convex body in $\mathbb{R}^{n}$, then

$$
\operatorname{Prob}\left(\left\{x \in K:\|x\|_{2} \geqslant c \sqrt{n} L_{K} t\right\}\right) \leqslant \exp (-\sqrt{n} t)
$$
\end{abstract}

for every $t \geqslant 1$, where $L_{K}$ denotes the isotropic constant.

\section{Introduction}

Let $K$ be an isotropic convex body in $\mathbb{R}^{n}$. This means that $K$ has volume equal to 1 , its centre of mass is at the origin and its inertia matrix is a multiple of the identity. Equivalently, there exists a positive constant $L_{K}$, the isotropic constant of $K$, such that

$$
\int_{K}\langle x, \theta\rangle^{2} d x=L_{K}^{2}
$$

for every $\theta \in S^{n-1}$. A major problem in Asymptotic Convex Geometry is whether there exists an absolute constant $c>0$ such that $L_{K} \leqslant c$ for every $n$ and every isotropic convex body $K$ in $\mathbb{R}^{n}$. The best known estimate, due to Bourgain (see [11]), is $L_{K} \leqslant c \sqrt[4]{n} \log n$, where $c>0$ is an absolute constant (see [30] for an extension of this estimate to the not-necessarily symmetric case). There is a number of recent developments on this problem; see [13], [14] and [21]. In particular, Klartag in [21] has obtained an isomorphic answer to the question: For every symmetric convex body $K$ in $\mathbb{R}^{n}$ there exists a second symmetric convex body $T$ in $\mathbb{R}^{n}$ whose Banach-Mazur distance from $K$ is $O(\log n)$ and its isotropic constant is bounded by an absolute constant: $L_{T} \leqslant c$.

${ }^{*}$ Research supported by a Marie Curie Intra-European Fellowship (EIF), Contract MEIF-CT-2005-025017. Part of this work was done while the author was a Postdoctoral Fellow at the University of Athens under the EPEAEK program "Pythagoras II". 
The starting point of this paper is the following concentration estimate of Alesker [1]: there exists an absolute constant $c>0$ such that if $K$ is an isotropic convex body in $\mathbb{R}^{n}$, then

$$
\operatorname{Prob}\left(\left\{x \in K:\|x\|_{2} \geqslant c \sqrt{n} L_{K} t\right\}\right) \leqslant 2 \exp \left(-t^{2}\right)
$$

for every $t \geqslant 1$.

Bobkov and Nazarov (see [7] and [8]) have clarified the picture of the volume distribution on isotropic unconditional convex bodies. Recall that a symmetric convex body $K$ is called unconditional if, for every choice of real numbers $t_{i}$ and every choice of signs $\varepsilon_{i} \in\{-1,1\}, 1 \leqslant i \leqslant n$,

$$
\left\|\varepsilon_{1} t_{1} e_{1}+\cdots+\varepsilon_{n} t_{n} e_{n}\right\|_{K}=\left\|t_{1} e_{1}+\cdots+t_{n} e_{n}\right\|_{K}
$$

where $\| \dot{\|}_{K}$ is the norm that corresponds to $K$ and $\left\{e_{1}, \ldots e_{n}\right\}$ is the standard orthonormal basis of $\mathbb{R}^{n}$. In particular, they obtained a striking strengthening of (1.2) in the case of 1-unconditional isotropic convex bodies: there exists an absolute constant $c>0$ such that if $K$ is a 1-unconditional isotropic convex body in $\mathbb{R}^{n}$, then

$$
\operatorname{Prob}\left(\left\{x \in K:\|x\|_{2} \geqslant c \sqrt{n} t\right\}\right) \leqslant \exp (-\sqrt{n} t)
$$

for every $t \geqslant 1$. Note that $L_{K} \simeq 1$ in the case of 1 -unconditional convex bodies (see [27]). Since the circumradius $R(K)$ of an isotropic convex body $K$ in $\mathbb{R}^{n}$ is always bounded by $(n+1) L_{K}$ (see [22]), the estimate in (1.3) is stronger than Alesker's estimate for all $t \geqslant 1$. It should be noted that similar very precise estimates on volume concentration were previously given in the case of the $\ell_{p}^{n}$-balls (see [39], [38], [41] and [40]). Volume concentration for the class of the unit balls of the Schatten trace classes was recently established in [19].

We will prove that an estimate similar to (1.3) holds true in full generality.

Theorem 1.1. There exists an absolute constant $c>0$ such that if $K$ is an isotropic convex body in $\mathbb{R}^{n}$, then

$$
\operatorname{Prob}\left(\left\{x \in K:\|x\|_{2} \geqslant c \sqrt{n} L_{K} t\right\}\right) \leqslant \exp (-\sqrt{n} t)
$$

for every $t \geqslant 1$.

The proof of Theorem 1.1 is based on the analysis of the growth of the $L_{q}$-norms

$$
I_{q}(K):=\left(\int_{K}\|x\|_{2}^{q} d x\right)^{1 / q}, \quad(1 \leqslant q \leqslant n)
$$

of the Euclidean norm $\|\cdot\|_{2}$ on isotropic convex bodies. It was observed in [32] that Theorem 1.1 follows from the following fact. 
Theorem 1.2. There exists an absolute constant $c>0$ with the following property: if $K$ is an isotropic convex body in $\mathbb{R}^{n}$, then

$$
I_{q}(K) \leqslant c \max \{q, \sqrt{n}\} L_{K}
$$

for every $2 \leqslant q \leqslant n$.

In fact, it was proved in [32] that Theorem 1.1 is equivalent to the fact that

$$
I_{q}(K) \leqslant c \sqrt{n} L_{K}
$$

for every $2 \leqslant q \leqslant \sqrt{n}$. An equivalent formulation of this last statement may be given in terms of the function

$$
f_{K}(t):=\int_{S^{n-1}}\left|K \cap\left(\theta^{\perp}+t \theta\right)\right| d \sigma(\theta) \quad(t \geqslant 0) .
$$

It has been conjectured that $f_{K}$ is close to the centered Gaussian density of variance $L_{K}^{2}$. This conjecture can be stated precisely in several different ways (see [10], [3]) and has been verified only for some special classes of bodies. It was proved in [32] that (1.7) is equivalent with the following:

Theorem 1.3. There exist absolute constants $c_{1}, c_{2}>0$ such that if $K$ is an isotropic convex body in $\mathbb{R}^{n}$, then

$$
f_{K}(t) \leqslant \frac{c_{1}}{L_{K}} \exp \left(-c_{2} \frac{t^{2}}{L_{K}^{2}}\right)
$$

for every $0<t \leqslant \sqrt[4]{n} L_{K}$.

The paper is organized as follows: In Section 2 we show how one can derive Theorem 1.1 from Theorem 1.2 (the argument appears in [32] and [33], but we reproduce it here so that the presentation will be self-contained). Our main tool is the study of the $L_{q}$-centroid bodies of $K$; the $q$-th centroid body $Z_{q}(K)$ has support function

$$
h_{Z_{q}(K)}(y)=\left(\int_{K}|\langle x, y\rangle|^{q} d x\right)^{1 / q} .
$$

Sections 3, 4 and 5 are devoted to an analysis of this family of bodies, which leads to Theorem 1.2. In fact, our method of proof works for an arbitrary convex body $K$ in $\mathbb{R}^{n}$, and leads to the following estimate:

Theorem 1.4. Let $K$ be a convex body in $\mathbb{R}^{n}$, with volume 1 and center of mass at the origin. Write $K$ in the form $K=T(\tilde{K})$, where $\tilde{K}$ is isotropic and $T \in S L(n)$ is positive definite. Then,

$$
\operatorname{Prob}\left(\left\{x \in K:\|x\|_{2} \geqslant c I_{2}(K) t\right\}\right) \leqslant \exp \left(-\frac{\|T\|_{H S}}{\lambda_{1}(T)} t\right)
$$

for every $t \geqslant 1$, where $c>0$ is an absolute constant (we write $\|T\|_{H S}$ for the Hilbert-Schmidt norm and $\lambda_{1}(T)$ for the largest eigenvalue of $T$ ). 
In other words, the concentration estimate of Theorem 1.1 is stable: if $\tilde{K}$ is isotropic and if $\|T\|_{H S} / \lambda_{1}(T)$ is not small, then one has strong concentration for $T(\tilde{K})$.

As a by-product of our method, in Section 6 we obtain a precise estimate for the volume of the $L_{q}$-centroid bodies of a convex body. The lower bound in the next Theorem is a consequence of the $L_{q}$ affine isoperimetric inequality of Lutwak, Yang and Zhang (see [26]).

Theorem 1.5. Let $K$ be a convex body in $\mathbb{R}^{n}$, with volume 1 and center of mass at the origin. For every $2 \leqslant q \leqslant n$ we have that

$$
c_{1} \sqrt{q / n} \leqslant\left|Z_{q}(K)\right|^{1 / n} \leqslant c_{2} \sqrt{q / n} L_{K},
$$

where $c_{1}, c_{2}>0$ are absolute constants.

In Section 7 we apply our concentration estimate to a question of Kannan, Lovász and Simonovits which has its origin in the problem of finding a fast algorithm for the computation of the volume of a given convex body: The isotropic condition (1.1) may be equivalently written in the form

$$
I=\frac{1}{L_{K}^{2}} \int_{K} x \otimes x d x
$$

where $I$ is the identity operator. Let $\varepsilon \in(0,1)$ and consider $N$ independent random points $x_{1}, \ldots, x_{N}$ uniformly distributed in $K$. The question is to find $N_{0}$, as small as possible, for which the following holds true: if $N \geqslant N_{0}$ then with probability greater than $1-\varepsilon$ one has

$$
\left\|I-\frac{1}{N L_{K}^{2}} \sum_{i=1}^{N} x_{i} \otimes x_{i}\right\|_{2} \leqslant \varepsilon .
$$

Kannan, Lovász and Simonovits (see [23]) proved that one can choose $N_{0}=c(\varepsilon) n^{2}$ for some constant $c(\varepsilon)>0$ depending only on $\varepsilon$. This was later improved to $N_{0} \simeq$ $c(\varepsilon) n(\log n)^{3}$ by Bourgain [12] and to $N_{0} \simeq c(\varepsilon) n(\log n)^{2}$ by Rudelson [36]. One can actually check (see [17]) that this last estimate can be obtained by Bourgain's argument if we also use Alesker's concentration inequality. See also [20] for an extension of this result. In [18] it was observed that $N_{0} \geqslant c(\varepsilon) n \log n$ is enough for the class of unconditional isotropic convex bodies. Theorem 1.1 allows us to prove the same fact in full generality.

Theorem 1.6. Let $\varepsilon \in(0,1)$. Assume that $n \geqslant n_{0}$ and let $K$ be an isotropic convex body in $\mathbb{R}^{n}$. If $N \geqslant c(\varepsilon) n \log n$, where $c>0$ is an absolute constant, and if $x_{1}, \ldots, x_{N}$ are independent random points uniformly distributed in $K$, then with probability greater than $1-\varepsilon$ we have

$$
(1-\varepsilon) L_{K}^{2} \leqslant \frac{1}{N} \sum_{i=1}^{N}\left\langle x_{i}, \theta\right\rangle^{2} \leqslant(1+\varepsilon) L_{K}^{2},
$$

for every $\theta \in S^{n-1}$. 
G. Aubrun has recently proved (see [2]) that in the unconditional case, only $C(\varepsilon) n$ random points are enough in order to obtain $(1+\varepsilon)$-approximation of the identity operator as in Theorem 1.6.

All the previous results remain valid if we replace Lebesgue measure on an isotropic convex body by an arbitrary isotropic log-concave measure. In the last Section of the paper, we briefly discuss this extension.

Notation. We work in $\mathbb{R}^{n}$, which is equipped with a Euclidean structure $\langle\cdot, \cdot\rangle$. We denote by $\|\cdot\|_{2}$ the corresponding Euclidean norm, and write $B_{2}^{n}$ for the Euclidean unit ball, and $S^{n-1}$ for the unit sphere. Volume is denoted by $|\cdot|$. We write $\omega_{n}$ for the volume of $B_{2}^{n}$ and $\sigma$ for the rotationally invariant probability measure on $S^{n-1}$. The Grassmann manifold $G_{n, k}$ of $k$-dimensional subspaces of $\mathbb{R}^{n}$ is equipped with the Haar probability measure $\mu_{n, k}$.

A convex body is a compact convex subset $C$ of $\mathbb{R}^{n}$ with non-empty interior. We say that $C$ is symmetric if $x \in C \Rightarrow-x \in C$. We say that $C$ has centre of mass at the origin if $\int_{C}\langle x, \theta\rangle d x=0$ for every $\theta \in S^{n-1}$. The support function $h_{C}: \mathbb{R}^{n} \rightarrow \mathbb{R}$ of $C$ is defined by $h_{C}(x)=\max \{\langle x, y\rangle: y \in C\}$. The gauge function $r_{C}: \mathbb{R}^{n} \rightarrow \mathbb{R}$ of $C$ is defined by $r_{C}(x)=\min \{\lambda \geqslant 0: x \in \lambda C\}$. The mean width of $C$ is defined as $2 w(C)$, where

$$
w(C)=\int_{S^{n-1}} h_{C}(\theta) \sigma(d \theta) .
$$

The circumradius of $C$ is the quantity $R(C)=\max \left\{\|x\|_{2}: x \in C\right\}$, and the polar body $C^{\circ}$ of $C$ is

$$
C^{\circ}:=\left\{y \in \mathbb{R}^{n}:\langle x, y\rangle \leqslant 1 \text { for all } x \in C\right\} .
$$

Whenever we write $a \simeq b$, we mean that there exist absolute constants $c_{1}, c_{2}>0$ such that $c_{1} a \leqslant b \leqslant c_{2} a$. The letters $c, c^{\prime}, c_{1}, c_{2}$ etc. denote absolute positive constants which may change from line to line. We refer to the books [37], [28] and [34] for basic facts from the Brunn-Minkowski theory and the asymptotic theory of finite dimensional normed spaces.

\section{Reduction to the behavior of moments}

Let $K$ be a convex body of volume 1 in $\mathbb{R}^{n}$. For every $q \geqslant 1$ we consider the $q$-th moment of the Euclidean norm

$$
I_{q}(K)=\left(\int_{K}\|x\|_{2}^{q} d x\right)^{1 / q}
$$

and, for every $q \geqslant 1$ and $y \in \mathbb{R}^{n}$, we set

$$
I_{q}(K, y)=\left(\int_{K}|\langle x, y\rangle|^{q} d x\right)^{1 / q} .
$$

Recall that, as a consequence of Borell's lemma (see [28, Appendix III]) one has the following Khintchine-type inequalities. 
Lemma 2.1. Let $K$ be a convex body of volume 1 in $\mathbb{R}^{n}$. For every $y \in \mathbb{R}^{n}$ and every $p, q \geqslant 1$ we have that

$$
I_{p q}(K, y) \leqslant c_{1} q I_{p}(K, y)
$$

where $c_{1}>0$ is an absolute constant. In particular, for every $y \in \mathbb{R}^{n}$ and every $q \geqslant 2$ we have that

$$
I_{q}(K, y) \leqslant\left(c_{1} / 2\right) q I_{2}(K, y)
$$

Also, for every $p, q \geqslant 1$ we have that

$$
I_{p q}(K) \leqslant c_{1} q I_{p}(K) .
$$

Alesker's concentration estimate (1.2) is equivalent to the following statement.

Theorem 2.2 (Alesker [1]). Let $K$ be an isotropic convex body in $\mathbb{R}^{n}$. For every $q \geqslant 2$ we have that

$$
I_{q}(K) \leqslant c_{2} \sqrt{q} I_{2}(K)
$$

where $c_{2}>0$ is an absolute constant.

We will prove the following fact.

Theorem 2.3. There exist universal constants $c_{3}, c_{4}>0$ with the following property: if $K$ is an isotropic convex body in $\mathbb{R}^{n}$, then

$$
I_{q}(K) \leqslant c_{4} I_{2}(K)
$$

for every $q \leqslant c_{3} \sqrt{n}$.

Theorem 1.2 is a direct consequence of Theorem 2.3, Lemma 3.9 and Lemma 3.11. Also, in [32] it was proved that Theorem 1.1 is equivalent to the fact that the $q$-th moments of the Euclidean norm stay bounded (and equivalent to $I_{2}(K)$ ) for large values of $q$. For completeness we show how one can derive Theorem 1.1 from Theorem 2.3 .

Proof of Theorem 1.1. Let $K$ be an isotropic convex body in $\mathbb{R}^{n}$. Fix $q \geqslant 2$. Markov's inequality shows that

$$
\operatorname{Prob}\left(x \in K:\|x\|_{2} \geqslant e^{3} I_{q}(K)\right) \leqslant e^{-3 q} .
$$

From Borell's lemma (see [28, Appendix III]) we get

$$
\begin{aligned}
\operatorname{Prob}\left(x \in K:\|x\|_{2} \geqslant e^{3} I_{q}(K) s\right) & \leqslant\left(1-e^{-3 q}\right)\left(\frac{e^{-3 q}}{1-e^{-3 q}}\right)^{(s+1) / 2} \\
& \leqslant e^{-q s}
\end{aligned}
$$

for every $s \geqslant 1$. Choosing $q=c_{3} \sqrt{n}$, and using (2.7), we get

$$
\operatorname{Prob}\left(x \in K:\|x\|_{2} \geqslant c_{4} e^{3} I_{2}(K) s\right) \leqslant e^{-c_{3} \sqrt{n} s}
$$

for every $s \geqslant 1$. Since $K$ is isotropic, we have $I_{2}(K)=\sqrt{n} L_{K}$. This proves Theorem 1.1. 


\section{$3 \quad L_{q}$-centroid bodies}

Let $K$ be a convex body of volume 1 in $\mathbb{R}^{n}$. For $q \geqslant 1$ we define the $L_{q}$-centroid body $Z_{q}(K)$ of $K$ by its support function:

$$
h_{Z_{q}(K)}(y)=I_{q}(K, y):=\left(\int_{K}|\langle x, y\rangle|^{q} d x\right)^{1 / q} .
$$

Since $|K|=1$, it is easy to check that $Z_{1}(K) \subseteq Z_{p}(K) \subseteq Z_{q}(K) \subseteq Z_{\infty}(K)$ for every $1 \leqslant p \leqslant q \leqslant \infty$, where $Z_{\infty}(K)=\operatorname{conv}\{K,-K\}$.

Observe that $Z_{q}(K)$ is always symmetric, and $Z_{q}(T K)=T\left(Z_{q}(K)\right)$ for every $T \in S L(n)$ and $q \in[1, \infty]$. Also, if $K$ has its center of mass at the origin, then $Z_{q}(K) \supseteq c Z_{\infty}(K)$ for all $q \geqslant n$, where $c>0$ is an absolute constant.

$L_{q}$-centroid bodies have appeared in the literature under a different normalization. If $K$ is a convex body in $\mathbb{R}^{n}$ and $1 \leqslant q<\infty$, the body $\Gamma_{q}(K)$ was defined in [25] by

$$
h_{\Gamma_{q}(K)}(y)=\left(\frac{1}{c_{n, q}|K|} \int_{K}|\langle x, y\rangle|^{q} d x\right)^{1 / q},
$$

where

$$
c_{n, q}=\frac{\omega_{n+q}}{\omega_{2} \omega_{n} \omega_{q-1}} .
$$

In other words, $Z_{q}(K)=c_{n, q}^{1 / q} \Gamma_{q}(K)$ if $|K|=1$. The normalization in [25] is chosen so that $\Gamma_{q}\left(B_{2}^{n}\right)=B_{2}^{n}$ for every $q$. Lutwak, Yang and Zhang (see [26] and [15] for a different proof) have established the following $L_{q}$ affine isoperimetric inequality.

Theorem 3.1. Let $K$ be a convex body of volume 1 in $\mathbb{R}^{n}$. For every $q \geqslant 1$,

$$
\left|\Gamma_{q}(K)\right| \geqslant 1
$$

with equality if and only if $K$ is a centered ellipsoid of volume 1.

Now, for every $p, q \geqslant 1$ we define

$$
w_{p}\left(Z_{q}(K)\right)=\left(\int_{S^{n-1}} h_{Z_{q}(K)}^{p}(\theta) \sigma(d \theta)\right)^{1 / p} .
$$

Observe that $w_{1}\left(Z_{q}(K)\right)=w\left(Z_{q}(K)\right)$.

The $q$-th moments of the Euclidean norm on $K$ are related to the $L_{q}$-centroid bodies of $K$ through the following Lemma.

Lemma 3.2. Let $K$ be a convex body of volume 1 in $\mathbb{R}^{n}$. For every $q \geqslant 1$ we have that

$$
w_{q}\left(Z_{q}(K)\right)=a_{n, q} \sqrt{\frac{q}{q+n}} I_{q}(K)
$$

where $a_{n, q} \simeq 1$. 
Proof. For every $x \in \mathbb{R}^{n}$ we have (see [31])

$$
\left(\int_{S^{n-1}}|\langle x, \theta\rangle|^{q} \sigma(d \theta)\right)^{1 / q}=a_{n, q} \frac{\sqrt{q}}{\sqrt{q+n}}\|x\|_{2}
$$

where $a_{n, q} \simeq 1$. Since

$$
w_{q}\left(Z_{q}(K)\right)=\left(\int_{S^{n-1}} \int_{K}|\langle x, \theta\rangle|^{q} d x \sigma(d \theta)\right)^{1 / q},
$$

the Lemma follows.

Remark. It is not hard to check that $a_{n, 2}=\sqrt{(n+2) /(2 n)}$ and

$$
I_{2}(K)=\sqrt{n} w_{2}\left(Z_{2}(K)\right) .
$$

Definition 3.3. Let $C$ be a symmetric convex body in $\mathbb{R}^{n}$ and let $\|x\|_{C}$ be the norm induced on $\mathbb{R}^{n}$ by $C$. Set

$$
M(C)=\int_{S^{n-1}}\|\theta\|_{C} d \sigma(\theta) \quad \text { and } \quad b(C)=\max _{x \in S^{n-1}}\|x\|_{C} .
$$

More generally, for every $q \geqslant 1$ set

$$
M_{q}(C)=\left(\int_{S^{n-1}}\|\theta\|_{C}^{q} d \sigma(\theta)\right)^{1 / q} .
$$

Define $k_{*}(C)$ as the largest positive integer $k \leqslant n$ for which

$$
\mu_{n, k}\left(F \in G_{n, k}: \frac{1}{2} M(C)\|x\|_{2} \leqslant\|x\|_{C} \leqslant 2 M(C)\|x\|_{2}, \forall x \in F\right) \geqslant \frac{n}{n+k} .
$$

The critical dimension $k_{*}$ is completely determined by the global parameters $M$ and $b$.

Fact 3.4 (Milman-Schechtman [29]). There exist $c_{1}, c_{2}>0$ such that

$$
c_{1} n \frac{M(C)^{2}}{b(C)^{2}} \leqslant k_{*}(C) \leqslant c_{2} n \frac{M(C)^{2}}{b(C)^{2}}
$$

for every symmetric convex body $C$ in $\mathbb{R}^{n}$.

We will make essential use of the following result of Litvak, Milman and Schechtman [24]:

Fact 3.5. There exist $c_{1}, c_{2}, c_{3}>0$ such that for every symmetric convex body $C$ in $\mathbb{R}^{n}$ we have:

(i) If $1 \leqslant q \leqslant k_{*}(C)$ then $M(C) \leqslant M_{q}(C) \leqslant c_{1} M(C)$.

(ii) If $k_{*}(C) \leqslant q \leqslant n$ then $c_{2} \sqrt{q / n} b(C) \leqslant M_{q}(C) \leqslant c_{3} \sqrt{q / n} b(C)$. 
On observing that $M\left(C^{\circ}\right)=w(C)$ and $b\left(C^{\circ}\right)=R(C)$, we can translate Fact 3.5 as follows:

Lemma 3.6. There exist $c_{1}, c_{2}, c_{3}>0$ such that for every symmetric convex body $C$ in $\mathbb{R}^{n}$ we have:

(i) If $1 \leqslant q \leqslant k_{*}\left(C^{\circ}\right)$ then $w(C) \leqslant w_{q}(C) \leqslant c_{1} w(C)$.

(ii) If $k_{*}\left(C^{\circ}\right) \leqslant q \leqslant n$ then $c_{2} \sqrt{q / n} R(C) \leqslant w_{q}(C) \leqslant c_{3} \sqrt{q / n} R(C)$.

Definition 3.7. Let $K$ be a convex body of volume 1 in $\mathbb{R}^{n}$. We define

$$
q_{*}(K)=\max \left\{q \in \mathbb{N}: k_{*}\left(Z_{q}^{\circ}(K)\right) \geqslant q\right\},
$$

where $Z_{q}^{\circ}(K):=\left(Z_{q}(K)\right)^{\circ}$.

We will need a lower estimate for $q_{*}(K)$. This depends on the " $\psi_{\alpha}$-behavior" of linear functionals on $K$.

Definition 3.8. Let $K$ be a convex body of volume 1 in $\mathbb{R}^{n}$ and let $\alpha \in[1,2]$. We say that $K$ is a $\psi_{\alpha}$-body with constant $b_{\alpha}$ if

$$
\left(\int_{K}|\langle x, \theta\rangle|^{q} d x\right)^{1 / q} \leqslant b_{\alpha} q^{1 / \alpha}\left(\int_{K}|\langle x, \theta\rangle|^{2} d x\right)^{1 / 2}
$$

for all $q \geqslant 2$ and all $\theta \in S^{n-1}$. Equivalently, if

$$
Z_{q}(K) \subseteq b_{\alpha} q^{1 / \alpha} Z_{2}(K)
$$

for all $q \geqslant 2$. Observe that if $K$ is a $\psi_{\alpha}$-body with constant $b_{\alpha}$, then $T(K)$ is a $\psi_{\alpha}$-body with the same constant, for every $T \in S L(n)$. Also, from (3.12) we see that

$$
R\left(Z_{q}(K)\right) \leqslant b_{\alpha} q^{1 / \alpha} R\left(Z_{2}(K)\right)
$$

for all $q \geqslant 2$.

An immediate consequence of Lemma 2.1 is that there exists an absolute constant $c>0$ such that every convex body $K$ in $\mathbb{R}^{n}$ is a $\psi_{1}$-body with constant $c$.

Lemma 3.9. There exist absolute constants $c_{1}, c_{2}>0$ such that if $K$ is a convex body of volume 1 in $\mathbb{R}^{n}$ then, for every $n \geqslant q \geqslant q_{*}(K)$,

$$
c_{1} R\left(Z_{q}(K)\right) \leqslant I_{q}(K) \leqslant c_{2} R\left(Z_{q}(K)\right) .
$$

In particular, if $K$ is an isotropic $\psi_{\alpha}$-body with constant $b_{\alpha}$ then, for every $n \geqslant q \geqslant$ $q_{*}(K)$,

$$
I_{q}(K) \leqslant c_{2} b_{\alpha} q^{1 / \alpha} L_{K}
$$


Proof. Let $n \geqslant q \geqslant q_{*}(K)$. By the definition of $q_{*}(K)$ we have $q \geqslant k_{*}\left(Z_{q}^{\circ}(K)\right)$, and Lemma 3.6(ii) shows that

$$
c_{3} \sqrt{\frac{q}{n}} R\left(Z_{q}(K)\right) \leqslant w_{q}\left(Z_{q}(K)\right) \leqslant c_{4} \sqrt{\frac{q}{n}} R\left(Z_{q}(K)\right) .
$$

Now, from Lemma 3.2 we have that

$$
w_{q}\left(Z_{q}(K)\right)=a_{n, q} \sqrt{\frac{q}{q+n}} I_{q}(K) .
$$

This proves (3.14). For the second assertion, we use (3.13) and the fact that $R\left(Z_{2}(K)\right)=L_{K}$ if $K$ is isotropic.

Remark. Let $K$ be a convex body in $\mathbb{R}^{n}$, with volume 1 and center of mass at the origin. If $q \geqslant n$, one can check that $R\left(Z_{q}(K)\right) \simeq I_{q}(K) \simeq R(K)$.

Proposition 3.10. There exists an absolute constant $c>0$ with the following property: if $K$ is a convex body of volume 1 in $\mathbb{R}^{n}$ which is $\psi_{\alpha}$-body with constant $b_{\alpha}$, then

$$
q_{*}(K) \geqslant c \frac{\left(k_{*}\left(Z_{2}^{\circ}(K)\right)\right)^{\alpha / 2}}{b_{\alpha}^{\alpha}} .
$$

In particular, for every convex body $K$ of volume 1 in $\mathbb{R}^{n}$ we have

$$
q_{*}(K) \geqslant c \sqrt{k_{*}\left(Z_{2}^{\circ}(K)\right)} .
$$

Proof. Let $q_{*}:=q_{*}(K)$. From Lemma 3.6(i), Lemma 3.2, Hölder's inequality and (3.6) we get

$$
\begin{aligned}
w\left(Z_{q_{*}}(K)\right) & \geqslant c_{1} w_{q_{*}}\left(Z_{q_{*}}(K)\right)=c_{1} a_{n, q_{*}} \sqrt{\frac{q_{*}}{n+q_{*}}} I_{q_{*}}(K) \\
& \geqslant c_{1} a_{n, q_{*}} \sqrt{\frac{q_{*}}{n+q_{*}}} I_{2}(K)=c_{1} a_{n, q_{*}} \sqrt{\frac{q_{*}}{n+q_{*}}} \sqrt{n} w_{2}\left(Z_{2}(K)\right) .
\end{aligned}
$$

In other words,

$$
w\left(Z_{q_{*}}(K)\right) \geqslant c_{2} \sqrt{q_{*}} w\left(Z_{2}(K)\right) .
$$

Since $K$ is a $\psi_{\alpha}$-body with constant $b_{\alpha}$, we have that

$$
R\left(Z_{q_{*}}(K)\right) \leqslant b_{\alpha} q_{*}{ }^{1 / \alpha} R\left(Z_{2}(K)\right) .
$$

Using the definition of $q_{*}$, Fact 3.4 and the inequalities (3.20) and (3.21), we write

$$
\begin{aligned}
q_{*}+1 & \geqslant k_{*}\left(Z_{q_{*}}^{\circ}(K)\right) \geqslant c_{3} n\left(\frac{w\left(Z_{q_{*}}(K)\right)}{R\left(Z_{q_{*}}(K)\right)}\right)^{2} \\
& \geqslant c_{3} n \frac{c_{2}^{2} q_{*}}{b_{\alpha}^{2} q_{*}^{2 / \alpha}} \frac{w^{2}\left(Z_{2}(K)\right)}{R^{2}\left(Z_{2}(K)\right)}=c_{5} \frac{q_{*}^{1-2 / \alpha}}{b_{\alpha}^{2}} k_{*}\left(Z_{2}^{\circ}(K)\right) .
\end{aligned}
$$


So, we get

$$
q_{*}(K) \geqslant c \frac{\left[k_{*}\left(Z_{2}^{\circ}(K)\right)\right]^{\alpha / 2}}{b_{\alpha}^{\alpha}} .
$$

The second assertion follows from the fact that every convex body is a $\psi_{1}$-body with (an absolute) constant $c>0$.

Observe that $K$ is isotropic if and only if $k_{*}\left(Z_{2}^{\circ}(K)\right)=n$. So, we get the following:

Corollary 3.11. There exists an absolute constant $c>0$ with the following property: if $K$ is an isotropic convex body of volume 1 in $\mathbb{R}^{n}$ which is $\psi_{\alpha}$-body with constant $b_{\alpha}$, then

$$
q_{*}(K) \geqslant \frac{c n^{\alpha / 2}}{b_{\alpha}^{\alpha}} .
$$

In particular, for every isotropic convex body $K$ in $\mathbb{R}^{n}$ we have that

$$
q_{*}(K) \geqslant c \sqrt{n} .
$$

\section{Projections of $L_{q}$-centroid bodies}

Let $K$ be a convex body of volume 1 in $\mathbb{R}^{n}$. Let $F \in G_{n, k}$ be a $k$-dimensional subspace of $\mathbb{R}^{n}$ and let $q \geqslant 1$. We define

$$
I_{q}(K, F)=\left(\int_{K}\left\|P_{F}(x)\right\|_{2}^{q} d x\right)^{1 / q},
$$

where $P_{F}$ denotes the orthogonal projection onto $F$, and

$$
w_{q}(K, F)=\left(\int_{S_{F}} h_{K}^{q}(\theta) d \sigma_{F}(\theta)\right)^{1 / q},
$$

where $S_{F}=S^{n-1} \cap F$ is the unit sphere of $F$. Observe that $w_{q}(K, F)=w_{q}\left(P_{F}(K)\right)$. We also set

$$
L(K, F)=\frac{I_{2}(K, F)}{\sqrt{k}}
$$

and

$$
L(K)=L\left(K, \mathbb{R}^{n}\right)=\frac{I_{2}(K)}{\sqrt{n}} .
$$

The argument we used for the proof of Lemma 3.2 shows that

$$
w_{q}\left(Z_{q}(K), F\right)=a_{k, q} \sqrt{\frac{q}{k+q}} I_{q}(K, F) .
$$


Choosing $q=2$ and taking into account (4.3) we get

$$
L^{2}(K, F)=\int_{S_{F}} h_{Z_{2}(K)}^{2}(\theta) d \sigma_{F}(\theta) .
$$

In particular, if $K$ is isotropic then

$$
L(K, F)=L(K)=L_{K}
$$

for every $F$.

In the sequel, we fix a $k$-dimensional subspace $F$ of $\mathbb{R}^{n}$ and denote by $E$ the orthogonal subspace of $F$. For every $\phi \in S_{F}$ we define $E(\phi)=\{x \in \operatorname{span}\{E, \phi\}$ : $\langle x, \phi\rangle \geqslant 0\}$.

Theorem 4.1 (K. Ball, see [4], [27]). Let $K$ a convex body of volume 1 in $\mathbb{R}^{n}$. For every $q \geqslant 0$ and $\phi \in F$, the function

$$
\phi \mapsto\|\phi\|_{2}^{1+\frac{q}{q+1}}\left(\int_{K \cap E(\phi)}|\langle x, \phi\rangle|^{q} d x\right)^{-\frac{1}{q+1}}
$$

is a gauge function on $F$.

Note. In [4] and [27], Theorem 4.1 is stated and proved for the case where $K$ is centrally symmetric. However, it was observed in [14] that the general case follows easily.

We denote by $B_{q}(K, F)$ the convex body in $F$ whose gauge function is defined in Theorem 4.1. The volume of $B_{q}(K, F)$ is given by

$$
\left|B_{q}(K, F)\right|=\omega_{k} \int_{S_{F}}\left(\int_{K \cap E(\phi)}|\langle x, \phi\rangle|^{q} d x\right)^{\frac{k}{q+1}} d \sigma_{F}(\phi) .
$$

To see this, express the volume of $B_{q}(K, F)$ in polar coordinates.

Lemma 4.2. Let $K$ be a convex body in $\mathbb{R}^{n}$. For every $q \geqslant 0$ and every $\theta \in S_{F}$, we have

$$
\int_{K}|\langle x, \theta\rangle|^{q} d x=k \omega_{k} \int_{S_{F}}|\langle\phi, \theta\rangle|^{q} \int_{K \cap E(\phi)}|\langle z, \phi\rangle|^{k+q-1} d z d \sigma_{F}(\phi) .
$$

Proof. For any continuous $f: \mathbb{R}^{n} \rightarrow \mathbb{R}$ we may write

$$
\begin{aligned}
\int_{K} f(x) d x & =\int_{E} \int_{F} \chi_{K}(u+v) f(u+v) d v d u \\
& =k \omega_{k} \int_{E} \int_{S_{F}} \int_{0}^{\infty} \chi_{K}(u+\rho \phi) f(u+\rho \phi) \rho^{k-1} d \rho d \sigma_{F}(\phi) d u \\
& =k \omega_{k} \int_{S_{F}}\left(\int_{E} \int_{0}^{\infty} \chi_{K}(u+\rho \phi) f(u+\rho \phi) \rho^{k-1} d \rho d u\right) d \sigma_{F}(\phi) .
\end{aligned}
$$


Observe that if $z=u+\rho \phi \in E(\phi)$ then $\rho=\langle z, \phi\rangle$. It follows that

$$
\int_{E} \int_{0}^{\infty} \chi_{K}(u+\rho \phi) f(u+\rho \phi) \rho^{k-1} d \rho d u=\int_{K \cap E(\phi)} f(z)\langle z, \phi\rangle^{k-1} d z .
$$

In other words,

$$
\int_{K} f(x) d x=k \omega_{k} \int_{S_{F}} \int_{K \cap E(\phi)} f(z)\langle z, \phi\rangle^{k-1} d z d \sigma_{F}(\phi) .
$$

Let $z \in K \cap E(\phi)$. Then $z=u+\langle\phi, z\rangle \phi$ for some $u \in E$, and hence, if $\theta \in F$ we have $\langle z, \theta\rangle=\langle\phi, \theta\rangle\langle z, \phi\rangle$. If we set $f_{\theta, q}(x)=|\langle x, \theta\rangle|^{q}$, then (4.11) becomes

$$
\int_{K}|\langle x, \theta\rangle|^{q} d x=k \omega_{k} \int_{S_{F}}|\langle\phi, \theta\rangle|^{q} \int_{K \cap E(\phi)}\langle z, \phi\rangle^{k+q-1} d z d \sigma_{F}(\phi) .
$$

This completes the proof of (4.9).

If we choose $q=0$ in (4.9), we can express the volume of $K$ in the following way:

$$
|K|=k \omega_{k} \int_{S_{F}} \int_{K \cap E(\phi)}|\langle x, \phi\rangle|^{k-1} d x d \sigma_{F}(\phi) .
$$

Notation. If $K$ is a convex body in $\mathbb{R}^{n}$, we set $\bar{K}=K /|K|^{1 / n}$; this is the dilation of $K$ which has volume 1 .

Proposition 4.3. Let $K$ be a convex body of volume 1 in $\mathbb{R}^{n}$ and let $1 \leqslant k \leqslant n-1$. For every $F \in G_{n, k}$ and every $q \geqslant 1$ we have that

$$
P_{F}\left(Z_{q}(K)\right)=(k+q)^{1 / q}\left|B_{k+q-1}(K, F)\right|^{1 / k+1 / q} Z_{q}\left(\bar{B}_{k+q-1}(K, F)\right) .
$$

Equivalently, for every $\theta \in F$,

$$
\int_{K}|\langle x, \theta\rangle|^{q} d x=(k+q) \int_{B_{k+q-1}(K, F)}|\langle x, \theta\rangle|^{q} d x .
$$

Proof. Let $\theta \in F$. Using polar coordinates on the right hand side of (4.15) and Lemma 4.2, we write

$$
\begin{aligned}
\int_{B_{k+q-1}(K, F)}|\langle x, \theta\rangle|^{q} d x & =\frac{k \omega_{k}}{k+q} \int_{S_{F}}|\langle\phi, \theta\rangle|^{q}\|\phi\|_{B_{k+q-1}(K, F)}^{-(k+q)} d \sigma_{F}(\phi) \\
& =\frac{k \omega_{k}}{k+q} \int_{S_{F}}|\langle\phi, \theta\rangle|^{q} \int_{K \cap E(\phi)}|\langle x, \phi\rangle|^{k+q-1} d x d \sigma_{F}(\phi) \\
& =\frac{1}{k+q} \int_{K}|\langle x, \theta\rangle|^{q} d x
\end{aligned}
$$

This proves (4.15). Observe that $h_{P_{F}\left(Z_{q}(K)\right)}(\theta)=h_{Z_{q}(K)}(\theta)$ for every $\theta \in F$. If we normalize the volume of $B_{k+q-1}(K, F)$, then (4.15) shows that

$$
h_{P_{F}\left(Z_{q}(K)\right)}(\theta)=(k+q)^{1 / q}\left|B_{k+q-1}(K, F)\right|^{1 / k+1 / q} h_{Z_{q}\left(\bar{B}_{k+q-1}(K, F)\right)}(\theta) .
$$

for every $\theta \in F$. This proves the Proposition. 
Notation. If $a, b$ are positive integers, we define

$$
B(b+1, a+1):=\int_{0}^{1} s^{a}(1-s)^{b} d s=\frac{a ! b !}{(a+b+1) !} .
$$

One may easily check that

$$
\left(\frac{b}{a}\right)^{a} \leqslant\left(\begin{array}{c}
b \\
a
\end{array}\right) \leqslant\left(\frac{e b}{a}\right)^{a}, \quad(0<a<b)
$$

and

$$
b^{a} \leqslant \frac{(a+b) !}{b !} \leqslant(a+b)^{a} .
$$

Let $n, k, q \in \mathbb{N}$, with $\max \{k, q\}<n$. We define

$$
A_{n, k, q}:=\left(\frac{B(n-k+1, k+q)^{\frac{k}{k+q}}}{B(n-k+1, k)}\right)^{\frac{k+q}{k q}} .
$$

Lemma 4.4. For every $n, k, q \in \mathbb{N}$, with $\max \{k, q\}<n$ we have that

$$
\frac{k^{\frac{1}{k}+\frac{1}{q}}}{(k+q)^{1 / q}} \frac{n}{n+q} \leqslant A_{n, k, q} \leqslant e \frac{k^{\frac{1}{k}+\frac{1}{q}}}{(k+q)^{1 / q}} \frac{k+q}{k} .
$$

Proof. We first write $A_{n, k, q}$ in the form

$$
A_{n, k, q}=\left(\frac{B(n-k+1, k+q)}{B(n-k+1, k)}\right)^{1 / q}(B(n-k+1, k))^{-1 / k} .
$$

Using (4.17) we can write

$$
\frac{B(n-k+1, k+q)}{B(n-k+1, k)}=\frac{k}{k+q} \frac{(k+q) !}{k !} \frac{n !}{(n+q) !}
$$

and

$$
(B(n-k+1, k))^{-1}=k\left(\begin{array}{l}
n \\
k
\end{array}\right) .
$$

Using (4.19) into (4.23) we get

$$
\frac{k}{k+q} \frac{k^{q}}{(n+q)^{q}} \leqslant \frac{B(n-k+1, k+q)}{B(n-k+1, k)} \leqslant \frac{k}{k+q} \frac{(k+q)^{q}}{n^{q}} .
$$

Using (4.18) into (4.24) we get

$$
k\left(\frac{n}{k}\right)^{k} \leqslant(B(n-k+1, k))^{-1} \leqslant k\left(\frac{e n}{k}\right)^{k} .
$$

Inserting (4.25) and (4.26) into (4.22) we get the Lemma.

The following lemma is standard and goes back at least to Berwald [5] (see [9] and [27]). 
Lemma 4.5. Let $C$ be a convex body in $\mathbb{R}^{m}$ and $0 \in \operatorname{int}(C)$. For every $\phi \in S^{m-1}$, set

$$
C_{+}(\phi):=\{x \in C:\langle x, \phi\rangle \geqslant 0\} .
$$

If $s \leqslant r$ are non-negative integers, we have that

$$
\left(\frac{\int_{C_{+}(\phi)}|\langle x, \phi\rangle|^{r} d x}{B(m, r+1)\left|C \cap \phi^{\perp}\right|}\right)^{1 /(r+1)} \leqslant\left(\frac{\int_{C_{+}(\phi)}|\langle x, \phi\rangle|^{s} d x}{B(m, s+1)\left|C \cap \phi^{\perp}\right|}\right)^{1 /(s+1)} .
$$

Proposition 4.6. Let $K$ be a convex body of volume 1 in $\mathbb{R}^{n}$ and $0 \in \operatorname{int}(K)$. If $F \in G_{n, k}$ and $E=F^{\perp}$ then, for every integer $q \geqslant 1$,

$$
\left|B_{k+q-1}(K, F)\right|^{\frac{1}{k}+\frac{1}{q}} \leqslant \frac{e(k+q)}{k}\left(\frac{1}{k+q}\right)^{\frac{1}{q}} \frac{1}{|K \cap E|^{1 / k}} .
$$

Proof. By (4.8) we have that

$$
\left|B_{k+q-1}(K, F)\right|=\omega_{k} \int_{S_{F}}\left(\int_{K \cap E(\phi)}|\langle x, \phi\rangle|^{k+q-1} d x\right)^{\frac{k}{k+q}} d \sigma_{F}(\phi) .
$$

Applying (4.28) with $C=K \cap \operatorname{span}\{E, \phi\}, m=n-k+1, r=k+q-1$ and $s=k-1$, we get

$$
\left(\frac{\int_{K \cap E(\phi)}|\langle x, \phi\rangle|^{k+q-1} d x}{B(n-k+1, k+q)|K \cap E|}\right)^{1 /(k+q)} \leqslant\left(\frac{\int_{K \cap E(\phi)}|\langle x, \phi\rangle|^{k-1} d x}{B(n-k+1, k)|K \cap E|}\right)^{1 / k}
$$

or, equivalently,

$$
\left(\int_{K \cap E(\phi)}|\langle x, \phi\rangle|^{k+q-1} d x\right)^{\frac{k}{k+q}} \leqslant \frac{A_{n, k, q}^{k q /(k+q)}}{(|K \cap E|)^{q /(k+q)}} \int_{K \cap E(\phi)}|\langle x, \phi\rangle|^{k-1} d x
$$

where $A_{n, k, q}$ is the constant defined by (4.20).

Going back to (4.30) and using (4.13) we get

$$
\begin{aligned}
\left|B_{k+q-1}(K, F)\right| & \leqslant \frac{A_{n, k, q}^{k q /(k+q)}}{|K \cap E|^{q /(k+q)}} \omega_{k} \int_{S_{F}} \int_{K \cap E(\phi)}|\langle x, \phi\rangle|^{k-1} d x d \sigma_{F}(\phi) \\
& =\frac{1}{k} \frac{A_{n, k, q}^{k q /(k+q)}}{|K \cap E|^{q /(k+q)}} .
\end{aligned}
$$

By Lemma 4.4 we conclude that

$$
\left|B_{k+q-1}(K, F)\right|^{\frac{1}{k}+\frac{1}{q}} \leqslant \frac{e(k+q)}{k}\left(\frac{1}{k+q}\right)^{\frac{1}{q}} \frac{1}{|K \cap E|^{1 / k}},
$$

as claimed. 
Lemma 4.7. Let $f_{1}, f_{2}: \mathbb{R}^{k} \rightarrow R$ be integrable functions with compact support such that $\int_{\mathbb{R}^{k}} f_{1}(x) d x=\int_{\mathbb{R}^{k}} f_{2}(x) d x$ and, for every $s>0, \int_{s B_{2}^{k}} f_{1}(x) d x \leqslant \int_{s B_{2}^{k}} f_{2}(x) d x$. Then, for every $p>0$,

$$
\int_{\mathbb{R}^{k}}\|x\|_{2}^{p} f_{1}(x) d x \geqslant \int_{\mathbb{R}^{k}}\|x\|_{2}^{p} f_{2}(x) d x .
$$

Proof. We write

$$
\begin{aligned}
\int_{\mathbb{R}^{k}}\|x\|_{2}^{p} f_{i}(x) d x & =\int_{\mathbb{R}^{k}} \int_{0}^{\|x\|_{2}} p s^{p-1} f_{i}(x) d s d x \\
& =\int_{0}^{\infty} p s^{p-1} \int_{\left(s B_{2}^{k}\right)^{c}} f_{i}(x) d x d s
\end{aligned}
$$

and observe that $\int_{\left(s B_{2}^{k}\right)^{c}} f_{1}(x) d x \geqslant \int_{\left(s B_{2}^{k}\right)^{c}} f_{2}(x) d x$ for every $s \geqslant 0$.

Proposition 4.8. Let $K$ be a convex body in $\mathbb{R}^{n}$, with volume 1 and center of mass at the origin. Let $F \in G_{n, k}$ and $E:=F^{\perp}$ Then

$$
\frac{1}{|K \cap E|^{1 / k}} \leqslant c L(K, F)
$$

where $c>0$ is an absolute constant.

Proof. Let $M:=\sup _{x \in F}|K \cap(E+x)|, f_{1}(x):=|K \cap(E+x)|$ and $f_{2}(x):=$ $M \chi_{\omega_{k}^{-1 / k} M^{-1 / k} B_{F}}(x)$, where $B_{F}=B_{2}^{n} \cap F$. Then,

$$
\int_{F} f_{1}(x) d x=1=\int_{F} f_{2}(x) d x
$$

and, from the fact that $f_{2}$ is equal to $M$ on a ball centered at the origin and equal to zero elsewhere, we easily check that

$$
\int_{s B_{F}} f_{1}(x) d x \leqslant \int_{s B_{F}} f_{2}(x) d x
$$

for every $s>0$. Lemma 4.7 shows that

$$
\int_{F}\|x\|_{2}^{2} f_{1}(x) d x \geqslant \int_{F}\|x\|_{2}^{2} f_{2}(x) d x=\frac{k}{k+2} \omega_{k}^{-2 / k} M^{-2 / k}=I_{2}^{2}\left(\bar{B}_{F}\right) M^{-2 / k} .
$$

Observe that

$$
\int_{F}\|x\|_{2}^{2} f_{1}(x) d x=\int_{K}\left\|P_{F} x\right\|_{2}^{2} d x=I_{2}^{2}(K, F)=k(L(K, F))^{2} .
$$

A result of Fradelizi (see [16]) shows that $M \leqslant e^{k}|K \cap E|$. This proves (4.35). 
Proposition 4.9. Let $K$ be a convex body in $\mathbb{R}^{n}$, with volume 1 and center of mass at the origin. If $F \in G_{n, k}$ and $E=F^{\perp}$ then, for every $q \in \mathbb{N}$ we have that

$$
P_{F}\left(Z_{q}(K)\right) \subseteq \frac{c(k+q)}{k} L(K, F) Z_{q}\left(\bar{B}_{k+q-1}(K, F)\right)
$$

where $c>0$ is an absolute constant.

Proof. We start from Proposition 4.3 and use Propositions 4.6 and 4.8 to estimate the quantity $\left|B_{k+q-1}(K, F)\right|^{1 / k+1 / q}$ which appears in (4.14).

Proposition 4.10. Let $K$ be a convex body in $\mathbb{R}^{n}$, with volume 1 and center of mass at the origin. For every $k$-dimensional subspace $F$ of $\mathbb{R}^{n}$ and every integer $q \geqslant 1$ there exists $\theta \in S_{F}$ such that

$$
h_{Z_{q}(K)}(\theta) \leqslant c \sqrt{k} \frac{k+q}{k} L(K, F),
$$

where $c>0$ is an absolute constant.

Proof. By Proposition 4.9, taking volumes in (4.40), we have that

$$
\left|P_{F}\left(Z_{q}(K)\right)\right|^{1 / k} \leqslant \frac{4 c(k+q)}{k} L(K, F)\left|Z_{q}\left(\bar{B}_{k+q-1}(K, F)\right)\right|^{1 / k} .
$$

Recall that

$$
\begin{aligned}
Z_{q}\left(\bar{B}_{k+q-1}(K, F)\right) & \subseteq \operatorname{conv}\left\{\bar{B}_{k+q-1}(K, F),-\bar{B}_{k+q-1}(K, F)\right\} \\
& \subseteq \bar{B}_{k+q-1}(K, F)-\bar{B}_{k+q-1}(K, F) .
\end{aligned}
$$

By the Rogers-Shephard inequality (see [35]) we have that

$$
\left|Z_{q}\left(\bar{B}_{k+q-1}(K, F)\right)\right|^{1 / k} \leqslant 4 .
$$

Therefore,

$$
\left|P_{F}\left(Z_{q}(K)\right)\right|^{1 / k} \leqslant \frac{4 c(k+q)}{k} L(K, F) .
$$

Assume that

$$
\rho\left(B_{2}^{n} \cap F\right) \subseteq P_{F}\left(Z_{q}(K)\right)
$$

for some $\rho>0$. The Proposition will be proved if we show that

$$
\rho \leqslant c \sqrt{k} \frac{k+q}{k} L(K, F) .
$$

From (4.44) and (4.45) we get

$$
\rho \omega_{k}^{1 / k} \leqslant \frac{4 c(k+q)}{k} L(K, F) .
$$

Since $\omega_{k}^{1 / k} \simeq 1 / \sqrt{k}$ we get $(4.41)$. 
Corollary 4.11. Let $K$ be a convex body in $\mathbb{R}^{n}$, with volume 1 and center of mass at the origin. For every integer $q \geqslant 1$ and every $F \in G_{n, q}$ there exists $\theta \in S_{F}$ such that

$$
h_{Z_{q}(K)}(\theta) \leqslant c \sqrt{q} L(K, F)
$$

where $c>0$ is an absolute constant.

\section{Proof of the main result}

We are now ready to give the proof of Theorem 2.3. The precise formulation of our result in the isotropic case is the following.

Theorem 5.1. There exists an absolute constant $c>0$ with the following property: if $K$ is an isotropic convex body in $\mathbb{R}^{n}$, then

$$
I_{q}(K) \leqslant c I_{2}(K)
$$

for every $q \leqslant q_{*}(K)$.

Proof. Set $q_{*}=q_{*}(K)$. By the definition of $q_{*}(K)$ and $k_{*}\left(Z_{q_{*}}^{\circ}(K)\right)$ we have $k_{*}\left(Z_{q_{*}}^{\circ}(K)\right) \geqslant q_{*}$, and hence, there exists a $q_{*}$-dimensional subspace $F$ of $\mathbb{R}^{n}$ such that

$$
h_{Z_{q_{*}}(K)}(\theta) \geqslant \frac{1}{2} w\left(Z_{q_{*}}(K)\right)
$$

for every $\theta \in S_{F}$.

On the other hand, Corollary 4.11 shows that there exists $\theta_{0} \in S_{F}$ such that

$$
h_{Z_{q_{*}}(K)}\left(\theta_{0}\right) \leqslant c_{1} \sqrt{q_{*}} L(K, F)=c_{1} \sqrt{q_{*}} L_{K},
$$

where $c_{1}>0$ is an absolute constant (here, we are using the fact that $K$ is isotropic; we have $L(K, F)=L_{K}$ for every subspace $F$ of $\left.\mathbb{R}^{n}\right)$. It follows that

$$
w\left(Z_{q_{*}}(K)\right) \leqslant 2 c_{1} \sqrt{q_{*}} L_{K} .
$$

Since $q_{*} \leqslant k_{*}\left(Z_{q_{*}}^{\circ}(K)\right)$, from Lemma 3.5 and Lemma 3.2 we have

$$
w\left(Z_{q_{*}}(K)\right) \geqslant c_{2} w_{q_{*}}\left(Z_{q_{*}}(K)\right) \geqslant c_{3} \sqrt{\frac{q_{*}}{n}} I_{q_{*}}(K) .
$$

Combining (5.4) and (5.5) we see that

$$
I_{q_{*}}(K) \leqslant c \sqrt{n} L_{K}
$$

for some absolute constant $c>0$. Since $\sqrt{n} L_{K}=I_{2}(K)$, the result follows.

Proof of Theorem 2.2. We have assumed that $K$ is isotropic. Then, Corollary 3.11 shows that $q_{*}(K) \geqslant c \sqrt{n}$, where $c>0$ is an absolute constant. Then, Theorem 2.2 is an immediate consequence of Theorem 5.1.

In fact, the method which has been developed in the previous Sections provides a similar result for an arbitrary convex body that has its center of mass at the origin: 
Theorem 5.2. Let $K$ be a convex body in $\mathbb{R}^{n}$, with volume 1 and center of mass at the origin. If $q_{*}=q_{*}(K)$, then

$$
I_{q_{*}}(K) \leqslant c I_{2}(K),
$$

where $c>0$ is an absolute constant.

For the proof of Theorem 5.2 we need one more Lemma.

Lemma 5.3. There exists a constant $c \in(0,1)$ with the following property: if $C$ is a symmetric convex body in $\mathbb{R}^{n}$ and if $m \leqslant k_{*}\left(C^{\circ}\right) \leqslant c n$, then

$$
w(C) \leqslant 2 \int_{B} \int_{S_{F}} h_{C}(\theta) d \sigma(\theta) d \mu_{n, m}(F),
$$

where

$$
B=\left\{F \in G_{n, m}: \frac{1}{2} w(C) \leqslant h_{C}(\theta) \leqslant 2 w(C) \text { for all } \theta \in S_{F}\right\} .
$$

Proof. Since $m \leqslant k_{*}\left(C^{\circ}\right)$, we have that $\mu_{n, m}\left(B^{c}\right) \leqslant \frac{m}{n+m}$, where $B^{c}=G_{n, m} \backslash B$. Then, using the fact that

$$
w_{2}(C) \leqslant c_{1} w(C)
$$

which can be easily checked from Lemma 3.6, we can write

$$
\begin{aligned}
w(C)= & \int_{G_{n, m}} \int_{S_{F}} h_{K}(\theta) d \sigma_{F}(\theta) d \mu_{n, m}(F) \\
= & \int_{B} \int_{S_{F}} h_{C}(\theta) d \sigma_{F}(\theta) d \mu_{n, m}(F)+\int_{B^{c}} \int_{S_{F}} h_{C}(\theta) d \sigma_{F}(\theta) d \mu_{n, m}(F) \\
\leqslant & \int_{B} \int_{S_{F}} h_{C}(\theta) d \sigma_{F}(\theta) d \mu_{n, m}(F) \\
& +\left(\mu\left(B^{c}\right)\right)^{1 / 2}\left(\int_{G_{n, m}}\left(\int_{S_{F}} h_{C}(\theta) d \sigma_{F}(\theta)\right)^{2} d \mu_{n, m}(F)\right)^{1 / 2} \\
\leqslant & \int_{B} \int_{S_{F}} h_{C}(\theta) d \sigma_{F}(\theta) d \mu_{n, m}(F) \\
& +\left(\mu\left(B^{c}\right)\right)^{1 / 2}\left(\int_{G_{n, m}} \int_{S_{F}} h_{C}^{2}(\theta) d \sigma_{F}(\theta) d \mu_{n, m}(F)\right)^{1 / 2} \\
\leqslant & \int_{B} \int_{S_{F}} h_{C}(\theta) d \sigma_{F}(\theta) d \mu_{n, m}(F)+\sqrt{\frac{m}{n+m}} w_{2}(C) \\
\leqslant & \int_{B} \int_{S_{F}} h_{C}(\theta) d \sigma_{F}(\theta) d \mu_{n, m}(F)+c_{1} \sqrt{\frac{m}{n+m}} w(C) \\
\leqslant & \int_{B} \int_{S_{F}} h_{C}(\theta) d \sigma_{F}(\theta) d \mu_{n, m}(F)+\frac{1}{2} w(C),
\end{aligned}
$$


provided that $c \in(0,1)$ is chosen small enough.

Proof of Theorem 5.2. We define

$$
q=\min \left\{q_{*},\lfloor c n\rfloor\right\}
$$

where $c \in(0,1)$ is the constant from Lemma 5.3. By Lemma 3.2 and Lemma 3.6 we get

$$
I_{q}(K)=a_{n, q}^{-1} \sqrt{\frac{q+n}{q}} w_{q}\left(Z_{q}(K)\right) \leqslant c_{1} a_{n, q}^{-1} \sqrt{\frac{q+n}{q}} w\left(Z_{q}(K)\right) .
$$

Set

$$
B=\left\{F \in G_{n, q}: \frac{1}{2} w\left(Z_{q}(K)\right) \leqslant h_{Z_{q}(K)}(\theta) \leqslant 2 w\left(Z_{q}(K)\right) \text { for all } \theta \in S_{F}\right\} .
$$

From Lemma 5.3 we have that

$$
w\left(Z_{q}(K)\right) \leqslant 2 \int_{B} \int_{S_{F}} h_{Z_{q}(K)}(\theta) d \sigma(\theta) d \mu_{n, q}(F) .
$$

Now, Corollary 4.11 and the definition of $B$ show that, for every $F \in B$, there exists $\theta_{0} \in S_{F}$ such that

$$
w\left(Z_{q}(K)\right) \leqslant 2 h_{Z_{q}(K)}\left(\theta_{0}\right) \leqslant 2 c_{2} \sqrt{q} L(K, F) .
$$

Using again the definition of $B$, we now see that for every $F \in B$ and for every $\theta \in S_{F}$ we have that

$$
h_{Z_{q}(K)}(\theta) \leqslant 2 w\left(Z_{q}(K)\right) \leqslant 4 c_{2} \sqrt{q} L(K, F) .
$$

In view of (4.6) this means that, for every $F \in B$ and for every $\theta \in S_{F}$,

$$
h_{Z_{q}(K)}(\theta) \leqslant 2 w\left(Z_{q}(K)\right) \leqslant 4 c_{2} \sqrt{q}\left(\int_{S_{F}} h_{Z_{2}(K)}^{2}(\phi) d \sigma_{F}(\phi)\right)^{1 / 2} .
$$

Going back to (5.14) we may write

$$
\begin{aligned}
w\left(Z_{q}(K)\right) & \leqslant 8 c_{2} \sqrt{q} \int_{B} \int_{S_{F}}\left(\int_{S_{F}} h_{Z_{2}(K)}^{2}(\phi) d \sigma_{F}(\phi)\right)^{1 / 2} d \sigma_{F}(\theta) d \mu_{n, q}(F) \\
& =8 c_{2} \sqrt{q} \int_{B}\left(\int_{S_{F}} h_{Z_{2}(K)}^{2}(\phi) d \sigma_{F}(\phi)\right)^{1 / 2} d \mu_{n, q}(F) \\
& \leqslant 8 c_{2} \sqrt{q}\left(\int_{G_{n, q}} \int_{S_{F}} h_{Z_{2}(K)}^{2}(\phi) d \sigma_{F}(\phi) d \mu_{n, q}(F)\right)^{1 / 2} \\
& =8 c_{2} \sqrt{q} L(K) .
\end{aligned}
$$


Then, (5.12) becomes

$$
I_{q}(K) \leqslant c_{1} a_{n, q}^{-1} \sqrt{\frac{q+n}{q}} \cdot\left(8 c_{2} \sqrt{q} L(K)\right) \leqslant c_{3} \sqrt{n} L(K) .
$$

Since $\sqrt{n} L(K)=I_{2}(K)$ by definition (see (4.4)), we finally get

$$
I_{q}(K) \leqslant c_{3} I_{2}(K)
$$

From Lemma 2.1 we know that

$$
I_{s}(K) \leqslant c_{4} \frac{s}{p} I_{p}(K)
$$

for all $s \geqslant p \geqslant 1$, where $c_{4}>0$ is an absolute constant, and hence, we can compare $I_{q_{*}}(K)$ with $I_{q}(K)$. This completes the proof.

Corollary 5.4. Let $K$ be an isotropic convex body in $\mathbb{R}^{n}$, which is $\psi_{a}$-body with constant $b_{\alpha}$. Then,

$$
I_{q}(K) \leqslant c \max \left\{b_{\alpha} q^{1 / \alpha}, \sqrt{n}\right\} L_{K}
$$

for every $2 \leqslant q \leqslant n$, where $c>0$ is an absolute constant. In particular, for every isotropic convex body $K$ in $\mathbb{R}^{n}$ we have that

$$
I_{q}(K) \leqslant c_{1} \max \{q, \sqrt{n}\} L_{K}
$$

for every $2 \leqslant q \leqslant n$, where $c_{1}>0$ is an absolute constant.

Proof. Direct consequence of Theorem 5.1 and Lemma 3.9.

It is interesting to note that the Euclidean ball and the $\ell_{1}^{n}$-ball $B_{1}^{n}$ are the extremal bodies in Theorem 5.2, in the following sense:

Proposition 5.5. Let $K$ be a convex body of volume 1 in $\mathbb{R}^{n}$. For every $0<p<$ $q<\infty$ we have that

$$
\frac{I_{q}(K)}{I_{p}(K)} \geqslant \frac{I_{q}\left(\overline{B_{2}^{n}}\right)}{I_{p}\left(\overline{B_{2}^{n}}\right)} .
$$

Proof. We follow an argument of Bobkov-Koldobsky from [6]. Let $0<p<q<\infty$. A simple computation shows that

$$
I_{p}^{p}\left(\overline{B_{2}^{n}}\right)=n \omega_{n} \int_{0}^{\omega_{n}^{-1 / n}} r^{n+p-1} d r=\frac{n}{n+p} \omega_{n}^{-p / n} .
$$

Therefore,

$$
\frac{I_{q}\left(\overline{B_{2}^{n}}\right)}{I_{p}\left(\overline{B_{2}^{n}}\right)}=\frac{\left(\frac{n}{n+q}\right)^{1 / q}}{\left(\frac{n}{n+p}\right)^{1 / p}}
$$


For every $q>-n$ we have that

$$
I_{q}^{q}(K)=\omega_{n} \int_{0}^{\infty} r^{n+q-1} \sigma\left(\frac{1}{r} K\right) d r
$$

The function $g(r)=\omega_{n} \sigma\left(\frac{1}{r} K\right)$ is non-increasing on $(0, \infty)$ and can be assumed absolutely continuous. So, we can write

$$
g(r)=n \int_{r}^{\infty} \frac{\rho(s)}{s^{n}} d s, \quad(r>0)
$$

for some non-negative function $\rho$ on $(0, \infty)$. Then,

$$
1=\int_{0}^{\infty} r^{n-1} g(r) d r=n \int_{0}^{\infty} \int_{0<r<s} r^{n-1} \frac{\rho(s)}{s^{n}} d r d s=\int_{0}^{\infty} \rho(s) d s .
$$

Hence, $\rho$ represents a probability density of a positive random variable, say, $\xi$. We now write

$$
I_{q}^{q}(K)=\int_{0}^{\infty} r^{q+n-1} g(r) d r=\frac{n}{n+q} \int_{0}^{\infty} s^{q} \rho(s) d s=\frac{n}{n+q} \mathbb{E}\left(\xi^{q}\right) .
$$

Applying Hölder's inequality for $0<p \leqslant q \leqslant \infty$, we see that

$$
\left(\mathbb{E}\left(\xi^{q}\right)\right)^{1 / q} \geqslant\left(\mathbb{E}\left(\xi^{p}\right)\right)^{1 / p}
$$

So,

$$
\frac{I_{q}(K)}{I_{p}(K)}=\frac{\left(\frac{n}{n+q} \mathbb{E}\left(\xi^{q}\right)\right)^{1 / q}}{\left(\frac{n}{n+p} \mathbb{E}\left(\xi^{p}\right)\right)^{1 / p}} \geqslant \frac{\left(\frac{n}{n+q}\right)^{1 / q}}{\left(\frac{n}{n+p}\right)^{1 / p}}=\frac{I_{q}\left(\overline{B_{2}^{n}}\right)}{I_{p}\left(\overline{B_{2}^{n}}\right)}
$$

as claimed.

We now pass to the $\ell_{1}^{n}$-ball; the results of [39] show that

$$
I_{q}\left(\overline{B_{1}^{n}}\right) \simeq \max \{q, \sqrt{n}\} L_{\overline{B_{1}^{n}}}
$$

for every $2 \leqslant q \leqslant n$. We will prove something more general:

Lemma 5.6. Let $K$ be an isotropic convex body in $\mathbb{R}^{n}$. Then, for every $1 \leqslant q \leqslant n$ we have

$$
I_{q}(K) \geqslant \frac{c q}{n} R(K)
$$

where $c>0$ is an absolute constant. 
Proof. From the Remark after Lemma 3.9 we know that for every convex body $K$ of volume 1 with center of mass at the origin, $R(K) \leqslant c_{1} I_{n}(K)$, where $c_{1}>0$ is an absolute constant. Also, Lemma 2.1 shows that, for every $p, q \geqslant 1$,

$$
I_{p q}(K) \leqslant c_{2} p I_{q}(K)
$$

where $c_{2}>0$ is an absolute constant.

Let $1 \leqslant q \leqslant n$. Then,

$$
R(K) \leqslant c_{1} I_{n}(K) \leqslant c_{1} c_{2} \frac{n}{q} I_{q}(K) .
$$

This proves the Lemma, with $c:=\frac{1}{c_{1} c_{2}}$.

Remark. Since $R\left(\overline{B_{1}^{n}}\right) \simeq n L_{\overline{B_{1}^{n}}}$, Lemma 5.6 and (5.22) prove (5.32).

Corollary 5.7. There exists an absolute constant c $>0$ such that for every isotropic convex body $K$ in $\mathbb{R}^{n}$ and for every $2 \leqslant q \leqslant \infty$,

$$
\frac{I_{q}\left(\overline{B_{2}^{n}}\right)}{I_{2}\left(\overline{B_{2}^{n}}\right)} \leqslant \frac{I_{q}(K)}{I_{2}(K)} \leqslant c \frac{I_{q}\left(\overline{B_{1}^{n}}\right)}{I_{2}\left(\overline{B_{1}^{n}}\right)} .
$$

Proof of Theorem 1.4. Let $K$ be an isotropic convex body in $\mathbb{R}^{n}$. If $T \in S L(n)$ is positive definite, then

$$
I_{2}^{2}(T(K))=\int_{T(K)}\|x\|_{2}^{2} d x=\int_{K}\left\langle x,\left(T^{*} T\right)(x)\right\rangle d x=\operatorname{tr}\left(T^{*} T\right) L_{K}^{2},
$$

by the isotropicity of $K$. Since $I_{2}(K)=\sqrt{n} w_{2}\left(Z_{2}(T(K))\right)$ and $\operatorname{tr}\left(T^{*} T\right)=\|T\|_{H S}^{2}$, we get

$$
w_{2}\left(Z_{2}(T(K))\right)=\frac{\|T\|_{H S}}{\sqrt{n}} L_{K}
$$

On the other hand,

$$
R\left(Z_{2}(T(K))\right)=R\left(T\left(Z_{2}(K)\right)\right)=R\left(T\left(L_{K} B_{2}^{n}\right)\right)=L_{K} R\left(T\left(B_{2}^{n}\right)\right)=L_{K} \lambda_{1}(T),
$$

where $\lambda_{1}(T)$ is the largest eigenvalue of $T$. It follows that

$$
k_{*}\left(Z_{2}^{\circ}(T K)\right) \simeq n\left(\frac{w\left(Z_{2}(T(K))\right)}{R\left(Z_{2}(T(K))\right)}\right)^{2} \simeq\left(\frac{\|T\|_{H S}}{\lambda_{1}(T)}\right)^{2} .
$$

From Proposition 3.10 we know that $q_{*}(T(K)) \geqslant c \sqrt{k_{*}\left(Z_{2}^{\circ}(K)\right)}$, and hence, Theorem 5.2 and the reduction scheme of Section 2 show that

$$
\operatorname{Prob}\left(\left\{x \in K:\|x\|_{2} \geqslant c I_{2}(K) t\right\}\right) \leqslant \exp \left(-\frac{\|T\|_{H S}}{\lambda_{1}(T)} t\right)
$$

for every $t \geqslant 1$, where $c>0$ is an absolute constant, which is the assertion of Theorem 1.4. 


\section{Volume of $L_{q}$-centroid bodies}

The $L_{q}$-affine isoperimetric inequality of Lutwak, Yang and Zhang (see Theorem 3.1) can be written in the following form.

Proposition 6.1. Let $K$ be a convex body in $\mathbb{R}^{n}$, with volume 1 and center of mass at the origin. Then,

$$
\left|Z_{q}(K)\right|^{1 / n} \geqslant\left|Z_{q}\left(\overline{B_{2}^{n}}\right)\right|^{1 / n} \geqslant c \sqrt{q / n}
$$

for every $1 \leqslant q \leqslant n$, where $c>0$ is an absolute constant.

Our goal in this Section is to show that the reverse inequality holds true (up to the isotropic constant).

Theorem 6.2. Let $K$ be a convex body in $\mathbb{R}^{n}$, with volume 1 and center of mass at the origin. For every $2 \leqslant q \leqslant n$ we have that

$$
\left|Z_{q}(K)\right|^{1 / n} \leqslant c \sqrt{q / n} L_{K}
$$

where $c>0$ is an absolute constant.

For the proof we will use the Aleksandrov-Fenchel inequalities for the quermassintegrals of a convex body $C$. From the classical Steiner's formula we know that

$$
\left|C+t B_{2}^{n}\right|=\sum_{k=0}^{n}\left(\begin{array}{l}
n \\
k
\end{array}\right) W_{[k]}(C) t^{k}
$$

for all $t>0$, where $W_{[k]}(C)$ is the $k$-th quermassintegral of $C ; W_{[k]}(C)$ is the mixed volume $V_{n-k}(C)=V\left(C ; n-k, B_{2}^{n} ; k\right)$.

The Aleksandrov-Fenchel inequality implies the log-concavity of the sequence $\left(W_{[0]}(C), \ldots, W_{[n]}(C)\right)$. In other words,

$$
W_{[j]}^{k-i}(C) \geqslant W_{[i]}^{k-j}(C) W_{[k]}^{j-i}(C), \quad(0 \leqslant i<j<k \leqslant n) .
$$

Choosing $k=n$ we see that

$$
\left(\frac{W_{[i]}(C)}{\omega_{n}}\right)^{1 /(n-i)} \leqslant\left(\frac{W_{[j]}(C)}{\omega_{n}}\right)^{1 /(n-j)}
$$

for all $1 \leqslant i<j<n$.

We will also use Kubota's integral formula which connects the $i$-th quermassintegral with the average of the volumes of the $(n-i)$-dimensional projections of $C$ :

$$
W_{[i]}(C)=\frac{\omega_{n}}{\omega_{n-i}} \int_{G_{n, n-i}}\left|P_{F}(C)\right| d \mu_{n, n-i}(F), \quad(1 \leqslant i \leqslant n-1) .
$$


Proof of Theorem 6.2. We may assume that $K$ is isotropic. It is enough to prove (6.2) for $q \in \mathbb{N}$ and $1 \leqslant q \leqslant n-1$.

Taking $k=q$ in (4.45) we see that

$$
\left|P_{F}\left(Z_{q}(K)\right)\right|^{1 / q} \leqslant c_{1} L_{K}
$$

where $c_{1}>0$ is an absolute constant. Applying (6.6) we get

$$
W_{[n-q]}\left(Z_{q}(K)\right) \leqslant \frac{\omega_{n}}{\omega_{q}}\left(c_{1} L_{K}\right)^{q} .
$$

Now, we apply (6.5) for $C=Z_{q}(K)$ with $j=n-q$ and $i=0$; this gives

$$
W_{[n-q]}^{n}\left(Z_{q}(K)\right) \geqslant\left|Z_{q}(K)\right|^{q} \omega_{n}^{n-q}
$$

or, equivalently,

$$
W_{[n-q]}^{1 / q}\left(Z_{q}(K)\right) \geqslant\left|Z_{q}(K)\right|^{1 / n} \omega_{n}^{1 / q-1 / n} .
$$

Combining (6.8) and (6.10) we get

$$
\left|Z_{q}(K)\right|^{1 / n} \leqslant \frac{\omega_{n}^{1 / n}}{\omega_{q}^{1 / q}} c L_{K}
$$

Since $\omega_{k}^{1 / k} \simeq 1 / \sqrt{k}$, the result follows.

\section{Random points in isotropic symmetric convex bodies}

For the proof of Theorem 1.6 we follow the argument of [18] which incorporates the concentration estimate of Theorem 1.1 into Rudelson's approach to the problem. The main lemma in [36] is the following.

Theorem 7.1 (Rudelson). Let $x_{1}, \ldots, x_{N}$ be vectors in $\mathbb{R}^{n}$ and let $\varepsilon_{1}, \ldots, \varepsilon_{N}$ be independent Bernoulli random variables which take the values \pm 1 with probability $1 / 2$. Then, for all $p \geqslant 1$,

$$
\left(\mathbb{E}\left\|\sum_{i=1}^{N} \varepsilon_{i} x_{i} \otimes x_{i}\right\|^{p}\right)^{1 / p} \leqslant c \sqrt{p+\log n} \cdot \max _{i \leqslant N}\left\|x_{i}\right\|_{2} \cdot\left\|\sum_{i=1}^{N} x_{i} \otimes x_{i}\right\|^{1 / 2},
$$

where $c>0$ is an absolute constant.

Proof of Theorem 1.6. Let $\varepsilon \in(0,1)$ and let $p \geqslant 1$. We first estimate the expectation of $\max _{i \leqslant N}\left\|x_{i}\right\|_{2}^{2 p}$, where $x_{1}, \ldots, x_{N}$ are independent random points uniformly distributed in $K$. 
Lemma 7.2. There exists $c>0$ such that for every isotropic convex body $K$ in $\mathbb{R}^{n}$, for every $N \in \mathbb{N}$ and every $p \geqslant 1$,

$$
\left(\mathbb{E} \max _{i \leqslant N}\left\|x_{i}\right\|_{2}^{p}\right)^{1 / p} \leqslant c L_{K} \max \{\sqrt{n}, p, \log N\}
$$

Proof. From Theorem 1.1 we have

$$
\operatorname{Prob}\left(x \in K:\|x\|_{2} \geqslant c q L_{K}\right) \leqslant \exp (-q)
$$

for every $q \geqslant \sqrt{n}$, where $c>0$ is an absolute constant. We set $A:=\max \{p, \sqrt{n}, \log N\}$. Since $A \geqslant \sqrt{n}$, we may write

$$
\begin{aligned}
\mathbb{E} \max _{i \leqslant N}\left\|x_{i}\right\|_{2}^{p} & =\int_{0}^{\infty} p t^{p-1} \operatorname{Prob}\left(\max _{i \leqslant N}\left\|x_{i}\right\|_{2} \geqslant t\right) d t \\
& \leqslant c^{p} L_{K}^{p} \int_{0}^{A} p t^{p-1} d t+p c^{p} L_{K}^{p} N \int_{A}^{\infty} t^{p-1} \operatorname{Prob}\left(\|x\|_{2} \geqslant c t L_{K}\right) d t \\
& \leqslant c^{p} L_{K}^{p} A^{p}+p c^{p} L_{K}^{p} N \int_{A}^{\infty} t^{p-1} e^{-t} d t \\
& \leqslant c^{p} L_{K}^{p} A^{p}+p c^{p} L_{K}^{p} N e^{-A+1} A^{p} \\
& \leqslant c^{p} L_{K}^{p} A^{p}\left(1+e p N e^{-A}\right) \\
& \leqslant c^{p} L_{K}^{p} A^{p}(1+e p)
\end{aligned}
$$

where we have used the fact that

$$
\int_{A}^{\infty} t^{p-1} e^{-t} d t \leqslant e^{-A+1} A^{p}
$$

for all $A \geqslant p \geqslant 1$.

Following Rudelson's argument (see also [18], page 10) we see that if $x_{1}^{\prime}, \ldots, x_{N}^{\prime}$ are independent random points from $K$ which are chosen independently from the $x_{i}$ 's, then

$$
S^{p}:=\mathbb{E}\left\|I-\frac{1}{N L_{K}^{2}} \sum_{i=1}^{N} x_{i} \otimes x_{i}\right\|^{p} \leqslant(4 c)^{p} \frac{(p+\log n)^{p / 2}}{N^{p / 2} L_{K}^{p}}\left(\mathbb{E} \max _{i \leqslant N}\left\|x_{i}\right\|_{2}^{2 p}\right)^{1 / 2} \sqrt{S^{p}+1} .
$$

If we choose $p=\log n$, Lemma 7.2 and (7.5) show that

$$
S^{p} \leqslant\left(\frac{c_{1}(\log n) \max \left\{n,(\log N)^{2}\right\}}{N}\right)^{p / 2} \sqrt{S^{p}+1} .
$$

From this inequality we see that if $N \geqslant c(\varepsilon) n \log n$ then

$$
\left(\frac{c_{1}(\log n) \max \left\{n,(\log N)^{2}\right\}}{N}\right)^{p / 2}<\frac{\varepsilon^{p+1}}{2},
$$


and hence,

$$
\mathbb{E}\left\|I-\frac{1}{N L_{K}^{2}} \sum_{i=1}^{N} x_{i} \otimes x_{i}\right\|^{p}=S^{p}<\varepsilon^{p+1} .
$$

An application of Markov's inequality shows that

$$
\operatorname{Prob}\left(\left\|I-\frac{1}{N L_{K}^{2}} \sum_{i=1}^{N} x_{i} \otimes x_{i}\right\|>\varepsilon\right)<\varepsilon,
$$

which is exactly the assertion of Theorem 1.6.

\section{Concluding Remarks}

All the main results of this paper remain valid if we replace Lebesgue measure on an isotropic convex body by an arbitrary isotropic log-concave measure. In our discussion, the fact that $K$ is a convex body was only used through the logconcavity of the function $t \rightarrow|\{x \in K:|\langle x, \theta\rangle|=t\}|$. Also our assumption that $K$ has centre of mass at the origin was needed in order to use Fradelizi's Theorem which is also valid for any log-concave probability mesure. One way to extend our results to the case of a $\log$-concave probability measure in $\mathbb{R}^{n}$ is to introduce the relevant parameters and follow the proofs of the previous Sections:

Let $\mu$ be a log-concave probability measure in $\mathbb{R}^{n}$. We say that $\mu$ has its center of mass at the origin if $\int_{\mathbb{R}^{n}}\langle x, \theta\rangle d \mu(x)=0$ for all $\theta \in S^{n-1}$. For $q \geqslant 1$ we define $I_{q}(\mu):=\left(\int_{\mathbb{R}^{n}}\|x\|_{2}^{q} d \mu(x)\right)^{1 / q}$ and we consider the symmetric convex body $Z_{q}(\mu)$ in $\mathbb{R}^{n}$ which has support function $h_{Z_{q}(\mu)}(\theta):=\left(\int_{\mathbb{R}^{n}}|\langle x, \theta\rangle|{ }^{q} d \mu(x)\right)^{1 / q}$.

Next, we define

$$
q_{*}(\mu)=\max \left\{q \in \mathbb{N}: k_{*}\left(Z_{q}^{\circ}(\mu)\right) \geqslant q\right\} .
$$

Then, one can prove the following analogue of Theorem 5.2:

Theorem 8.1. Let $\mu$ be a log-concave probability measure in $\mathbb{R}^{n}$ with center of mass at the origin. Then, for every $q \leqslant q_{*}(\mu)$,

$$
I_{q}(\mu) \leqslant c I_{2}(\mu)
$$

where $c>0$ is an absolute constant.

The proof of Theorem 8.1 is similar to the proof of Theorem 5.2; only a few straightforward modifications are needed.

Let $\mu$ be a log-concave probability measure in $\mathbb{R}^{n}$. We say that $\mu$ is isotropic if $Z_{2}(\mu)$ is a multiple of the Euclidean ball. An inspection of the proofs in Section 3 makes it clear that Proposition 3.10 and Corollary 3.11 remain true in the "logconcave" case. This implies immediately a reformulation of Theorem 2.3 for logconcave measures. 
Acknowledgements: I thank M. Fradelizi, A. Giannopoulos, O. Guédon and A. Pajor for helpful discussions.

\section{References}

[1] S. Alesker, $\psi_{2}$-estimate for the Euclidean norm on a convex body in isotropic position, Geom. Aspects of Funct. Analysis (Lindenstrauss-Milman eds.), Oper. Theory Adv. Appl. 77 (1995), 1-4.

[2] G. Aubrun, Sampling convex bodies: a random matrix approach, Preprint.

[3] M. Anttila, K.M. Ball and I. Perissinaki, The central limit problem for convex bodies, Trans. Amer. Math. Soc. 355 (2003), 4723-4735.

[4] K. M. Ball, Logarithmically concave functions and sections of convex sets in $\mathbb{R}^{n}$, Studia Math. 88 (1988), 69-84.

[5] L. Berwald, Verallgemeinerung eines Mittelswertsatzes von J.Favard, für positive konkave Funkionen., Acta Math. 79 (1947), 17-37.

[6] S. G. Bobkov and A. Koldobsky, On the central limit property of convex bodies, Geom. Aspects of Funct. Analysis (Milman-Schechtman eds.), Lecture Notes in Math. 1807 (2003), 44-52.

[7] S. G. Bobkov and F. L. Nazarov, On convex bodies and log-concave probability measures with unconditional basis, Geom. Aspects of Funct. Analysis (MilmanSchechtman eds.), Lecture Notes in Math. 1807 (2003), 53-69.

[8] S. G. Bobkov and F. L. Nazarov, Large deviations of typical linear functionals on a convex body with unconditional basis, Stochastic Inequalities and Applications, Progr. Probab. 56, Birkhauser, Basel (2003), 3-13.

[9] C. Borell, Complements of Lyapunov's inequality, Math. Ann. 205 (1973), 323-331.

[10] U. Brehm and J. Voigt, Asymptotics of cross sections for convex bodies, Beiträge Algebra Geom. 41 (2000), 437-454.

[11] J. Bourgain, On the distribution of polynomials on high dimensional convex sets, Geom. Aspects of Funct. Analysis (Lindenstrauss-Milman eds.), Lecture Notes in Math. 1469 (1991), 127-137.

[12] J. Bourgain, Random points in isotropic convex bodies, in Convex Geometric Analysis (Berkeley, CA, 1996) Math. Sci. Res. Inst. Publ. 34 (1999), 53-58.

[13] J. Bourgain, On the isotropy constant for $\psi_{2}$-bodies, Geom. Aspects of Funct. Analysis (Milman-Schechtman eds.), Lecture Notes in Math. 1807 (2003), 114-121.

[14] J. Bourgain, B. Klartag and V. D. Milman, Symmetrization and isotropic constants of convex bodies, Geom. Aspects of Funct. Analysis (Milman-Schechtman eds.), Lecture Notes in Math. 1850 (2004), 101-115.

[15] S. Campi and P. Gronchi, The $L^{p}$-Busemann-Petty centroid inequality, Adv. in Math. 167 (2002), 128-141.

[16] M. Fradelizi, Sections of convex bodies through their centroid, Arch. Math. 69 (1997), 515-522. 
[17] A. Giannopoulos and V. D. Milman, Concentration property on probability spaces, Adv. in Math. 156 (2000), 77-106.

[18] A. Giannopoulos, M. Hartzoulaki and A. Tsolomitis, Random points in isotropic unconditional convex bodies, J. London Math. Soc. 72 (2005), 779-798.

[19] O. Guédon and G. Paouris, Concentration of mass on the Schatten classes, Ann. IHP Probab. Stat. (to appear).

[20] O. Guédon and M. Rudelson, $L_{p}$ moments of random vectors via majorizing measures, Preprint.

[21] B. Klartag, An isomorphic version of the slicing problem, J. Funct. Anal. 218 (2005), no. $2,372-394$.

[22] R. Kannan, L. Lovász and M. Simonovits, Isoperimetric problems for convex bodies and a localization lemma, Discrete Comput. Geom. 13 (1995), 541-559.

[23] R. Kannan, L. Lovász and M. Simonovits, Random walks and $O^{*}\left(n^{5}\right)$ volume algorithm for convex bodies, Random Structures Algorithms II1 (1997), 1-50.

[24] A. Litvak, V.D. Milman and G. Schechtman, Averages of norms and quasi-norms, Math. Ann. 312 (1998), 95-124.

[25] E. Lutwak and G. Zhang, Blaschke-Santaló inequalities, J. Differential Geom. 47 (1997), 1-16.

[26] E. Lutwak, D. Yang and G. Zhang, $L^{p}$ affine isoperimetric inequalities, J. Differential Geom. 56 (2000), 111-132.

[27] V.D. Milman and A. Pajor, Isotropic position and inertia ellipsoids and zonoids of the unit ball of a normed n-dimensional space, Geom. Aspects of Funct. Analysis (Lindenstrauss-Milman eds.), Lecture Notes in Math. 1376 (1989), 64-104.

[28] V.D. Milman and G. Schechtman, Asymptotic Theory of Finite Dimensional Normed Spaces, Lecture Notes in Math. 1200 (1986), Springer, Berlin.

[29] V.D. Milman and G. Schechtman, Global versus Local asymptotic theories of finitedimensional normed spaces, Duke Math. Journal 90 (1997), 73-93.

[30] G. Paouris, On the isotropic constant of non-symmetric convex bodies, Geom. Aspects of Funct. Analysis (Milman-Schechtman eds.), Lecture Notes in Math. 1745 (2000), 238-243.

[31] G. Paouris, $\Psi_{2}$-estimates for linear functionals on zonoids, Geom. Aspects of Funct. Analysis (Milman-Schechtman eds.), Lecture Notes in Math. 1807 (2003), 211-222.

[32] G. Paouris, Concentration of mass and central limit properties of isotropic convex bodies, Proc. Amer. Math. Soc. 133 (2005), no. 2, 565-575.

[33] G. Paouris, On the $\Psi_{2}$-behavior of linear functionals on isotropic convex bodies, Studia Math. 168 (2005), no. 3, 285-299.

[34] G. Pisier, The Volume of Convex Bodies and Banach Space Geometry, Cambridge Tracts in Mathematics 94 (1989).

[35] C. A. Rogers and G. C. Shephard, The difference body of a convex body, Arch. Math. 8 (1957), 220-233.

[36] M. Rudelson, Random vectors in the isotropic position, J. Funct. Anal. 164 (1999), 60-72. 
[37] R. Schneider, Convex Bodies: The Brunn-Minkowski Theory, Encyclopedia of Mathematics and its Applications 44, Cambridge University Press, Cambridge (1993).

[38] G. Schechtman and M. Schmuckenschläger, Another remark on the volume of the intersection of two $L_{p}^{n}$ balls, Geom. Aspects of Funct. Analysis (Lindenstrauss-Milman eds.), Lecture Notes in Math. 1469, Springer, Berlin, 1991, 174-178.

[39] G. Schechtman and J. Zinn, On the volume of the intersection of two $L_{p}^{n}$ balls, Proc. Amer. Math. Soc. 110 (1990), 217-224.

[40] G. Schechtman and J. Zinn, Concentration on the $\ell_{p}^{n}$ ball, Geom. Aspects of Funct. Analysis (Milman-Schechtman eds.), Lecture Notes in Math. 1745, Springer, Berlin, 2000, 245-256.

[41] M. Schmuckenschläger, Volume of intersections and sections of the unit ball of $\ell_{p}^{n}$, Proc. Amer. Math. Soc. 126 (1998), 1527-1530

G. PAOuris: Équipe d'Analyse et de Mathématiques Appliquées, Université de Marne-laVallée, Champs sur Marne, 77454, Marne-la-Vallée, Cedex 2, France.

E-mail: grigoris_paouris@yahoo.co.uk

AdDed IN PRoOfs: B. Klartag has recently proved that for every convex body $K$ in $\mathbb{R}^{n}$ and for every $\varepsilon>0$ there exists a second convex body $T$ in $\mathbb{R}^{n}$ whose BanachMazur distance from $K$ is bounded by $1+\varepsilon$ and and its isotropic constant satisfies $L_{T} \leqslant C / \sqrt{\varepsilon}$. This almost isometric answer to the slicing problem, combined with Theorem 1.1 of our paper, leads to the estimate $L_{K} \leqslant c \sqrt[4]{n}$ for every convex body $K$. Klartag's work will appear in this Journal. 